geplante Konferenz über Globale Wasserpolitik (BE 34 und 36), zu beobachten.

(b) Die Ansprechpartner in einer erweiterten Partnerstruktur, d. h. unter Einbeziehung von Gebietskörperschaften, Privatsektor, NRO und Medien (vgl. BE 27), müssen systematisch erfasst, ihr spezifischer Informationsbedarf muss ermittelt werden.

(c) Die Informationspolitik muss nach dem Prinzip der Gleichwertigkeit aller drei Länder sowie in der Absicht erfolgen, durch präventive, proaktive Informationsarbeit unproduktive Konfliktkonstellationen zu verhindern bzw. abzuschwächen. Dafür sollten phasen- und zielgruppenspezifische Einzelmaßnahmen erarbeitet werden.

3. Kommunikationssystem

Das Informationssystem hat einen Abbau von Wissensasymmetrien durch die Verteilung gleichwertiger Informationen zum Ziel, sucht dies aber durch einen asymmetrischen Pro- zess (hin zur Zielgruppe) zu erreichen. Dagegen besteht die Aufgabe des Kommunikationssystems darin, Kommunikation auf gleicher Wissens- und Statusbasis herzustellen und den top down- durch einen bottom-up-Ansatz zu ergänzen bzw. $\mathrm{zu}$ ersetzen (vgl. BE 28).

Dafür sind folgende Gesichtspunkte relevant:

(a) Auswahl von Partnern unter Einbeziehung der o.g. erweiterten Partnerstruktur. Dabei muss der Kreis der Kommunikationspartner die am Konflikt beteiligten Akteure möglichst repräsentativ abbilden. Er muss aber schon aus praktischen Erwägungen heraus enger sein als der Kreis der durch das Informationssystem erfassten Akteure.

(b) Ermittlung von Zeitfenstern im Vorfeld als möglich erachteter Konflikte bzw. im Konfliktverlauf, die für bestimmte Maßnahmen der Konfliktregulierung bzw. der Abwendung als unproduktiv erachteter Konfliktkonstellationen geeignet erscheinen.

\title{
Dokumentation
}

\section{Außen- und sicherheitspolitische Programme der Bundestagsparteien 2002}

\section{Aus dem Regierungsprogramm der SPD 2002-2006}

1. Deutschlands Rolle in Europa und der Welt

Verantwortung übernehmen

Eine gerechte Weltordnung

Deutschland in Europa

Unsere Bundeswehr

\section{Verantwortung übernehmen}

Deutschlands Rolle in der Welt hat sich verändert. Unsere Stimme hat an Gewicht gewonnen. Unser Rat und unsere Hilfe sind gefragt. Die Staatengemeinschaft zählt auf Deutschland, und sie kann sich auf Deutschland verlassen. Dazu haben wir mit unserer Politik entscheidend beigetragen.

Weil wir Mitverantwortung tragen für die gemeinsame Sicherheit in unserer einen Welt, haben wir die Außenpolitik, die Politik der wirtschaftlichen Zusammenarbeit und die Sicherheitspolitik schrittweise weiter entwickelt. Diesen Prozess wollen wir fortsetzen und durch die Geltung gemeinsamer Rechtsgrundsätze weltweit stärken.

Unser Ziel ist die Sicherung des Friedens weltweit. Interessengegensätze müssen friedlich ausgeglichen werden.

Mit uns wird von Deutschland niemals Aggression ausgehen. Aber die Wirklichkeit der Welt ist nicht nur friedlich. Unsere Aufgabe besteht heute in der Prävention von Konflikten und der Eindämmung von Gewalt. Wir engagieren uns für eine aktive Friedenspolitik. Dabei ergibt sich unsere Rolle in dieser Politik aus der geografischen und politischen Lage Deutschlands im Herzen Europas, unserer Mitgliedschaft in der Europäischen Union, den Vereinten Nationen und als Partner im Atlantischen Bündnis. Unsere Friedenspolitik wird bestimmt durch unsere Werte und Überzeugungen und unsere nationalen Interessen.

$\mathrm{Ob}$ auf dem Balkan, bei der Neuordnung und dem Wiederaufbau in Afghanistan, bei der Lösung regionaler Konflikte oder im Kampf gegen den internationalen Terrorismus - seit 1998 war Deutschland auch sicherheitspolitisch verstärkt gefordert und ist seiner internationalen Verantwortung gerecht geworden. Und zwar so, wie es unserem Selbstverständnis entspricht und wie unsere Partner und die Staatengemeinschaft es von uns erwarten können.

Mit der staatlichen Einheit haben wir nicht nur unsere nationale Souveränität in vollem Umfang zurückgewonnen, sondern zugleich auch das Recht und die Pflicht zur internationalen Solidarität. Wir sind ein normales europäisches Land geworden.

Wir stehen zum Einsatz unserer Soldaten international, wo er durch Beschlüsse der Vereinten Nationen und des Deutschen Bundestages legitimiert und den Soldaten gegenüber verantwortbar ist. Man darf sich seiner Verantwortung nicht entziehen, wenn Völkermord oder die Eskalation gewaltsamer Konflikte drohen oder geschehen. Das ist auch eine Lehre aus unserer eigenen Geschichte.

\section{Die Vereinten Nationen stärken}

Frieden und die weltweite Achtung der Menschenrechte sind unser Ziel. Der Weg dorthin führt über verstärkte Zusammenarbeit, über aktive Hilfe bei der wirtschaftlichen Entwicklung und ein gemeinsames System der Sicherheit und des Rechts.

Wir haben den Menschen nicht nur Märkte anzubieten, sondern auch Chancen und Solidarität.

Die Vereinten Nationen sind unverzichtbar für die Zusammenarbeit auf dieser Welt. Die Mehrzahl der 189 Mitgliedsstaaten 
der Vereinten Nationen verfügt über keine andere Instanz zu ihrer Interessenvertretung.

Die Vereinten Nationen zu stärken und ihnen durch mehr Rechte und eine innere Reform bessere und erweiterte Arbeitsmöglichkeiten zu geben, bleibt deshalb unser Ziel.

Deutschland ist bereit, sich in den Vereinten Nationen noch stärker zu engagieren.

Die Anforderungen an Sicherheit sind vielfältiger, komplexer und weniger berechenbar geworden. Deutschland ist heute ein Partner für Sicherheit und Stabilität in Europa und der Welt und verfolgt eine konsequente Politik des Interessenausgleiches und des Multilateralismus.

Wir werden auch in Zukunft eine aktive Friedens- und Sicherheitspolitik voranbringen, die die Prävention - wie in Makedonien erfolgreich geschehen - in den Mittelpunkt der Außen-, Entwicklungs- und Sicherheitspolitik rückt, die Unteilbarkeit der Sicherheit anerkennt und auf die Zusammenarbeit über nationale und regionale Grenzen hinweg setzt. Prävention erfordert Unterstützung für politische Konfliktlösungen in den Regionen, für Rüstungskontrolle, Abrüstung und Vertrauensbildung, für wirtschaftliche Entwicklung, für sozialen Ausgleich. Eine umfassende und wirksame Friedenspolitik benötigt auch die Verfügbarkeit leistungsfähiger militärischer Fähigkeiten. Zu Beginn des 21. Jahrhunderts ist der Beitrag der Bundeswehr zu einer solchen Politik der Friedenssicherung noch wichtiger geworden.

Deutschland ist gefordert, wenn Vereinte Nationen, NATO, EU und OSZE sich zur Abwehr von Gefahren und zur Sicherung des Friedens engagieren und wird seine internationalen Verpflichtungen erfüllen. Dabei müssen unsere nationalen Interessen und Fähigkeiten beachtet werden.

Die Fortsetzung einer Politik der Abrüstung und Rüstungskontrolle leistet Beiträge zu einer vorausschauenden Friedenspolitik. $\mathrm{Zu}$ einer Weiterentwicklung der vertragsgestützten Abrüstungspolitik gibt es keine Alternative. Das Ziel der Nichtverbreitung von Massenvernichtungswaffen steht bei uns weiter an erster Stelle.

Die Verträge zur Non-Proliferation, das Chemiewaffenübereinkommen, das B-Waffen-Übereinkommen und das Regime der Nichtverbreitung von Trägerwaffentechnologie (MTCR) sind zu stärken. Der START-Prozess muss fortgesetzt und der Atomteststopp-Vertrag (CTBT) ratifiziert werden. (...)

\section{Deutschland in Europa}

Wir gehören zu Europa und Europa gehört zu uns. Deutschland mit seinen Regionen ist und bleibt dabei unsere Heimat. Das Zusammenwachsen Europas in der Europäischen Union ist weltweit eine der hoffnungsvollsten Entwicklungen überhaupt. Die Stabilität, die die europäische Integration gefunden hat, begründet Frieden und Wohlstand für unseren Kontinent dauerhaft. Jetzt stehen weitere wichtige Entscheidungen darüber an, wie es weitergeht in Europa und welches unverwechselbare Gesicht Europa bekommen soll. In diesem Jahrzehnt muss eine Erweiterung der EU gelingen, die vor allem die europäischen Länder des ehemals kommunistischen Herrschaftsbereichs einbezieht.
Und es muss die EU eine Gestaltungskraft entwickeln, die demokratisch legitimiert und politisch effizient - der neuen Aufgabe gerecht wird.

Die europäische Idee hat ihren eigenen Wert, aber sie ist auch die unverzichtbare Antwort auf die Globalisierung. Wir tun alles, den europäischen Integrationsprozess voranzubringen und dabei die wohlbegründeten deutschen Interessen zu berücksichtigen.

(...)

\section{Europäische Außen- und Sicherheitspolitik ausbauen}

Eine gemeinsame Außen- und Sicherheitspolitik der EU ist Voraussetzung für gleichberechtigte transatlantische Partnerschaft, für eine engere Zusammenarbeit Europas mit Russland und für ein abgestimmteres Auftreten der EU in internationalen Organisationen wie der OSZE und den Vereinten Nationen.

Eine stärkere Rolle der Europäer in der Allianz und eine stärkere sicherheitspolitische Rolle der EU wird die NATO stärken. Die transatlantische Partnerschaft bleibt die Grundlage europäischer Sicherheit und die NATO die entscheidende politische und institutionelle Klammer für die euro-atlantische Gemeinschaft.

Die europäische Union muss mit der GASP ein umfassendes Sicherheitskonzept entwickeln, das politische, militärische, wirtschaftliche, soziale und ökologische Elemente umfasst, die europäische Außen-, Sicherheits- und Verteidigungs- und Entwicklungspolitik verzahnt und die Fähigkeit zur Konfliktprävention verstärkt.

Künftig wird die EU auch militärisch selbständig im Krisenmanagement handeln können, wenn sich die NATO als Ganzes nicht engagiert. Eine gemeinsame europäische Grenzpolizei wird an den künftigen Außengrenzen der Europäischen Union für einen wirkungsvollen Schutz gegen organisierte Kriminalität und illegale Einwanderung sorgen.

(...)

\section{Unsere Bundeswehr}

Wir werden die Reform der Bundeswehr weiterführen. Sie richtet die Bundeswehr auf die Herausforderungen des 21. Jahrhunderts aus, passt die Organisation der Bundeswehr den neuen Anforderungen an und investiert zielgerichtet in Menschen und Ausrüstungen, beseitigt Ungleichgewichte in Personalstruktur und Besoldung, setzt auf moderne ManagementGrundsätze.

Mit dem Reformprozess werden Aufgabenumfang, Organisation, Ausrüstung und Mittel der Bundeswehr wieder in ein ausgewogenes Verhältnis gebracht und das Stationierungsund Standortkonzept bis 2006 abgeschlossen. Mit der mittelfristigen Finanzplanung für die Bundeswehr und dem beschlossenen Programm für Innere und Äußere Sicherheit haben wir finanzielle Planungssicherheit für die Bundeswehr geschaffen. Die materielle Ausstattung der Streitkräfte soll kontinuierlich verbessert werden.

Die allgemeine Wehrpflicht und die große Zahl von Zeitsoldaten, die nur für eine begrenzte Frist Dienst in den Streitkräften leisten und dann in alle Bereiche des zivilen Lebens zurückkehren, stellen sicher, dass die Bundeswehr künftig in der Gesellschaft fest verankert bleibt. Deshalb bleibt es bei der Wehrpflicht. 
Quelle: http://regierungsprogramm.spd.de/servlet/PB/menu/ 1076211/index.html.

\section{Aus dem Regierungsprogramm der CDU/CSU 2002-2006}

Vertrauen für Deutschland in Europa und in der Welt

Voraussetzung für Freiheit und Demokratie ist Sicherheit. Sicherheit und Freiheit für die Menschen in Deutschland können nur im Bündnis mit unseren Partnern und Freunden in Europa und in der Welt geschützt werden. Daran haben die schrecklichen Terroranschläge in New York und Washington am 11. September 2001 nachdrücklich erinnert.

\section{Europa muss man richtig machen}

Die Einigung Europas ist das wertvollste Erbe des 20. Jahrhunderts. Die europäische Einigung ist die zentrale Gestaltungsaufgabe, um Frieden und Freiheit dauerhaft zu sichern. Sie muss auf dem Vertrauen der Bürger und auf einer gründlichen öffentlichen Diskussion der Perspektiven für Europa aufbauen. Die Handlungsfähigkeit Europas muss gestärkt werden. Selbstbewusste Nationen und Regionen sind die Bausteine Europas. Ihre geschichtlich gewachsene Vielfalt ist Europas Stärke. Nur mit Föderalismus, Subsidiarität und klar abgegrenzten, kraftvoll wahrgenommenen Kompetenzen der Ebenen Kommune, Region, Nation und Europa kann eine gute Zukunft gestaltet werden.

Deutschland hat an Gewicht in Europa verloren. Die Regierung Schröder zeigt kein echtes Engagement für die europäische Einigung. Sie hat die kleinen Partnerstaaten, darunter vor allem Deutschlands Nachbarn Österreich, wiederholt vor den Kopf gestoßen. Der deutsch-französische Motor der Europäischen Integration stottert, die Beziehungen zu Frankreich wurden vernachlässigt.

(...)

Wir werden die EU-Osterweiterung erfolgreich zu Ende führen. Deutschland als Land in der Mitte Europas muss mit allen Nachbarn gleichermaßen partnerschaftlich verbunden sein. Es ist allerdings Aufgabe der amtierenden Bundesregierung, zu einem sachgerechten Abschluss der Beitrittsverhandlungen bis Ende 2002 den Beitrag Deutschlands zu leisten.

Die Osterweiterung der Europäischen Union eröffnet die historische Chance, Frieden, Freiheit und Sicherheit in ganz Europa nachhaltig zu sichern. Die Erweiterung der Europäischen Union wird diesseits und jenseits ihrer bisherigen Grenzen Märkte entwickeln, Arbeitsplätze sichern und den Menschen mehr Wohlstand bringen. Je solider die jetzt laufenden Beitrittsverhandlungen geführt werden, um so eher wird diese Chance genutzt.

(...)

Wir werden die in Ansätzen stecken gebliebene Reform der Europäischen Institutionen vorantreiben. Wir wollen die demokratische Legitimation der europäischen Institutionen stärken. Das Ziel, eine Union mit 25 oder mehr Mitgliedstaaten handlungsfähig zu halten, ist nicht erreicht. Europa braucht eine Kommission in arbeitsfähiger Größe. Für Gesetzgebung und Budget sollen Parlament und Rat gemeinsam zuständig sein. Dabei sollten die Sitze im Europäischen Parlament den Mitgliedstaaten - Ausnahme eine Mindestzahl für die kleinsten - grundsätzlich nach der Bevölkerungszahl zugeordnet werden. Der Rat soll seine Entscheidungen grundsätzlich mit gewichteter Mehrheit treffen. Voraussetzung für den Übergang zu weiteren Mehrheitsentscheidungen ist aber eine klare Kompetenzabgrenzung. Vertragsänderungen, die Aufnahme neuer Mitglieder, die Inanspruchnahme der Generalklauseln, die Steuerpolitik und die Einnahmen der Europäischen Union müssen dagegen in der Einstimmigkeit verbleiben.

(...)

Alle notwendigen grundsätzlichen Regelungen, insbesondere zur Verteilung der Zuständigkeiten zwischen der Europäischen Union und den Mitgliedstaaten, zur Finanzverfassung sowie zu den Institutionen der Europäischen Union und ihren Verfahrensweisen sollen mit der EU-Grundrechte-Charta in einem Verfassungsvertrag zusammengefasst werden.

\section{Transatlantische Partnerschaft stärken Gesamteuropäische Einigung fördern}

Im 21. Jahrhundert wird die deutsche Außenpolitik immer mehr von den Aufgaben und Chancen bestimmt, die sich aus der Globalisierung ergeben. Die Fortschritte in der Kommunikationstechnik sowie die große Mobilität von Wissen und Kapital bringen den Menschen mehr Freiheit. Offenheit und wachsende Vernetzung machen jedoch die einzelnen Staaten und die bestehenden Ordnungsstrukturen auch verwundbarer: Die hochtechnisierte freie Welt ist Angriffsziel weltweit operierender Terroristen. Im »globalen Dorf «, in dem die verschiedensten Nationen, Religionen und Traditionen immer stärker zusammenrücken, treten die kulturellen Unterschiede deutlicher zutage.

(...)

Wir werden Europa stärken, jedoch nicht in Konkurrenz zu Amerika, sondern mit dem Ziel, aus Europa einen verantwortungsfähigeren Partner bei der Wahrnehmung internationaler Sicherheitsaufgaben zu machen. Nur gemeinsam mit Amerika können wir den neuen Sicherheitsbedrohungen wirksam begegnen. Dafür muss der europäische Pfeiler der NATO gestärkt werden. Wir halten es für unerlässlich, der gemeinsamen Außen- und Sicherheitspolitik der EU auch gemeinsame Instrumentarien zur Verfügung zu stellen. Dies erfordert eine abgestimmte Struktur, Ausrüstung sowie militärische und politische Einsatzfähigkeit für die europäischen Streitkräfte. Nur dadurch können schrittweise die europäischen Fähigkeiten wieder mit den amerikanischen kompatibel werden. Die europäischen Krisenreaktionskräfte müssen endlich einsatzfähig werden. Wir brauchen eine europäische industrielle Basis. Die europäischen Krisenreaktionsverbände sind ein erster Schritt auf dem Weg zur weiteren Integration von europäischen Streitkräften. Wir streben deshalb im Rahmen der Europäischen Sicherheits- und Verteidigungspolitik (ESVP) eine gemeinsame Streitkräfteplanung, eine gemeinsame Rüstungspolitik sowie ein gemeinsames militärisches Beschaffungswesen an. Dies schafft zudem in Zeiten knapper Haushalte wichtige Synergien.

Wir werden die verlässliche Freundschaft zu Amerika pflegen, die transatlantische Partnerschaft stärken und deshalb ein ernst zu nehmender Partner sein. Die enge Verbindung zwischen Europa und den USA ist weltweit einzigartig: Auf der Basis gemeinsamer Werte können wir gemeinsame Antworten auf die weltpolitischen Herausforderungen finden. Wir werden uns für eine neue »Atlantische Charta« und mehr 
Zusammenarbeit in den Bereichen Strafverfolgung, Energie und Umwelt einsetzen. Eine vertiefte Zusammenarbeit der Nachrichtendienste wird zu einer gemeinsamen Einschätzung weltweiter Bedrohungen beitragen.

(...)

Aufgrund der zahlreichen Konflikte im Krisenbogen Balkan, Kaukasus, Nah-/Mittelost und nördliches Afrika werden wir gemeinsam mit unseren Partnern den strategischen Fokus der Atlantischen Allianz nach Südosten ausrichten. Auch deshalb müssen beim NATO-Gipfeltreffen im Herbst, soweit die jeweilige innenpolitische Lage es erlaubt, nicht nur Slowenien, die Slowakei, Estlands, Litauen und Lettland, sondern auch Bulgarien und Rumänien zum Beitritt eingeladen werden.

Gemeinsam mit unseren Partnern im Bündnis werden wir entschlossen gegen die weltweite Verbreitung von Massenvernichtungswaffen vorgehen. Wir werden die Pläne von Präsident Bush zur Schaffung einer »Alliierten Raketenabwehr« aktiv aufgreifen und uns mit eigenen Initiativen für eine europäische Schutzkomponente einbringen.

(...)

\section{Zukunft für die Bundeswehr}

Art und Herkunft der Bedrohungen für unsere Sicherheit haben sich seit dem Ende des Kalten Krieges wesentlich gewandelt, die Trennlinien zwischen äußerer und innerer Sicherheit verwischen zusehends. $\mathrm{Zu}$ den herkömmlichen Anforderungen an unsere Streitkräfte sind neue Aufgaben hinzugekommen. Doch gerade im Kampf gegen den internationalen Terrorismus wird auch die wachsende militärischtechnologische Lücke zwischen Europäern und Amerikanern offensichtlich. Was sich im Kosovo-Krieg 1999 bereits abzeichnete, hat sich inzwischen zu einer Gefährdung des militärischen Zusammenhalts innerhalb der Atlantischen Allianz ausgeweitet.

Die Bundeswehr muss wieder voll einsatz- und bündnisfähig werden. Dafür werden wir die notwendigen Korrekturen vornehmen und eine glaubwürdige Finanzierung sicherstellen. Denn die Bundeswehr macht heute eine ideelle, personelle und materielle Auszehrung durch, die zu kaum wiedergutzumachenden Langzeitschäden führt. Aufgaben, Struktur und Finanzen passen nicht mehr zusammen, die Bundeswehr leidet unter einer drastischen Unterfinanzierung. Die Lücke zwischen den internationalen Zusagen und den tatsächlichen Fähigkeiten klafft immer weiter auseinander. Die Europäische Sicherheits- und Verteidigungspolitik darf nicht wegen unzureichender Beiträge Deutschlands scheitern. Der deutsche Beitrag zu den in Helsinki vereinbarten europäischen Einsatzkräften muss endlich glaubhaft geleistet werden.

Wir werden wieder für ein volles Vertrauensverhältnis der Angehörigen der Bundeswehr in ihre politische Führung sorgen. Den Soldaten und zivilen Mitarbeitern muss wieder eine attraktive Perspektive sowie die bestmögliche Ausbildung und Ausrüstung gegeben werden. Die Bundeswehr als Institution muss wieder öffentliche Anerkennung finden. Im Unterschied zu Rot-Grün werden wir die Streitkräfte nicht länger als notwendiges Übel ansehen, sondern sie als unverzichtbaren Bestandteil eines souveränen Staates achten.

Deutschland steht nach Bevölkerung und Wirtschaftskraft im Bündnis der NATO an zweiter Stelle. Unser Beitrag zur gemeinsamen Sicherheit muss der Bedeutung unseres
Landes angemessen sein. Das Konzept der Bundeswehrreform muss ergänzt und die Bundeswehr in Wehrstruktur, Umfang und Ausrüstung so erneuert werden, dass sie ihren Auftrag erfüllen kann: Landes- und Bündnisverteidigung; die neuen Aufgaben der Prävention, der Krisenreaktion und des Stabilitätstransfers; den Beitrag ihrer spezifischen Fähigkeiten zur zivil-militärischen Zusammenarbeit im Innern. Eine zeitgemäße Bundeswehrreform baut auf einer Neuorientierung und Anpassung der Zielsetzung der deutschen Streitkräfte auf. Für die Zukunft wichtige Fähigkeiten sind: Rasche Einsatzfähigkeit schnell verlegbarer Verbände, personelle und materielle Durchhaltefähigkeit, höchste technische Standards bei der Bewaffnung, bei den Führungs- und Kommunikationsmitteln und bei der Aufklärung. Zur Abwehr der Bedrohung durch den internationalen Terrorismus sind mehr Antiterroreinheiten, Gebirgs- und Fallschirmjäger notwendig und auch eine Verstärkung der Kräfte zum Objektschutz, des Pionierwesens, des ABC-Schutzes und des Sanitätswesens. (...)

Quelle: http://www.cdu.de/regierungsprogramm/.

\section{Aus dem Wahlprogramm von Bündnis90/Die Grünen 2002- 2006}

\section{Europäische Außen- und Sicherheitspolitik}

Der Ausbau der europäischen Außen-, Sicherheits- und Verteidigungspolitik ist notwendig, um die Rolle der Europäischen Union bei der Förderung gemeinsamer Sicherheit und gerechten Friedens in der Welt zu stärken.

Angesichts neuer Aufrüstungsschübe muss die Zivilmacht Europa zugleich Vorreiter für Rüstungskontrolle und Abrüstung werden und vorrangig ihre Fähigkeiten zur nichtmilitärischen Krisenbewältigung ausbauen. Wir setzen auf eine Priorität ziviler Konfliktbearbeitungsinstrumente. Im Sinne der besseren demokratischen Kontrolle und Handlungsfähigkeit ist eine stärkere Rolle des Europäischen Parlaments und der Kommission in allen Bereichen der Außen- und Sicherheitspolitik erforderlich.

\section{Südosteuropa stabilisieren}

Südosteuropa ist seit dem Umbruch von 1989 die Krisenregion Europas. In keiner anderen Region der Welt ist Deutschland auf mittlere Sicht in vergleichbarer Weise in Konfliktprävention und Krisenbewältigung engagiert.

Die Perspektive der europäischen Integration ist mehr und mehr zum Ordnungsfaktor in Südosteuropa geworden. Die Fortführung des Stabilitätspaktes für Südosteuropa ist ein wichtiges Element, um die genzüberschreitende Kooperation in der Region zur Grundlage ihrer politischen und wirtschaftlichen Verflechtung zu machen.

\section{Dauerhafter Frieden}

Kriege zu verhindern, Gewalt einzudämmen und Frieden zu fördern ist und bleibt Kern unserer Politik. Deshalb wollen wir, dass Prävention Vorrang hat. Wir machen uns stark für die von Kofi Annan geforderte »Kultur der Prävention« und den »Dialog der Kulturen«. Dazu gehört das Bemühen, zur Entspannung regionaler Konflikte, z. B. im Nahen und Mittleren Osten, beizutragen. Dazu zählt, die nationalen und internationalen Fähigkeiten der Zivilen Konfliktbearbeitung 
zu stärken und ihren Rückstand gegenüber den militärischen Fähigkeiten aufzuholen.

Angemessene Frühwarn- und Analysekapazitäten benötigen eine bessere Personalausstattung des Auswärtigen Amtes und wachsende Förderung der Friedensforschung. Sollen Friedensmissionen von UN und OSZE wirksam sein, brauchen sie mehr qualifiziertes und schnell verfügbares Fachpersonal. Die Schlüsselaufgabe internationaler Polizeimissionen ist ohne zusätzliches qualifiziertes Personal bei Bund und Ländern nicht mehr zu bewältigen. Um gesellschaftliche Verständigungsprozesse in Krisenregionen nicht nur punktuell, sondern in der Breite zu fördern, müssen Fachkräfte und Projekte des Zivilen Friedensdienstes und anderer gesellschaftlicher Akteure in den nächsten Jahren vervielfacht werden.

Der systematische Aufbau der Infrastruktur für zivile Konfliktbearbeitung braucht einen Aktionsplan, stetig wachsende Friedensinvestitionen und auch regionale Ausbildungsstätten (z. B. Friedensakademien).

\section{Die Vereinten Nationen stärken}

Aus unserer Sicht bilden die Vereinten Nationen den Hauptrahmen für eine weltweite Ordnungspolitik, bei der die Menschenrechte im Zentrum stehen. Deshalb wollen wir die UN als wichtigste internationale Institution zur Lösung globaler Probleme und Konflikte stärken. Wir wollen das Völkerrecht im Rahmen der Charta der Vereinten Nationen weiterentwickeln. Dazu gehört auch eine Reform des Sanktionsregimes der UN. Der Sicherheitsrat muss reformiert werden. Die angemessene Repräsentanz der Staaten muss auch im Sicherheitsrat gewährleistet sein. Die Handlungsfähigkeit der Vereinten Nationen und ihrer Einzelorganisationen muss gefördert werden.

Angesichts der Zunahme von Konflikten sind die Anforderungen an die UN dabei wesentlich komplexer geworden. Wir werden uns deshalb nachdrücklich für den Ausbau von Ansätzen regionaler Sicherheitskooperation in der OSZE und in außereuropäischen Regionen einsetzen, um die Fähigkeiten und Instrumente zur Konfliktprävention in den Krisenregionen selbst zu stärken. Wir unterstützen alle Bemühungen, die dazu führen, dass der Internationale Strafgerichtshof sich zu einer unabhängigen und wirkungsvollen globalen Gerichtsbarkeit entwickeln kann.

\section{Bundeswehr reformieren - die Wehrpflicht beenden}

Aufgabe der Bundeswehr ist, zur Gewalteindämmung und -vorbeugung im Dienste gemeinsamer Sicherheit beizutragen und im Rahmen des Bündnisses Sicherheit gegenüber äußeren militärischen Bedrohungen zu gewährleisten. Einsätze der Bundeswehr sind nach klaren und engen Kriterien zu entscheiden. Dazu gehört ein eindeutiges Mandat der Vereinten Nationen. Militärinterventionen zu Gunsten von Machtinteressen lehnen wir ab. Der Bundestag muss jeden Einzelfall prüfen und entscheiden. Ein »Entsendegesetz« lehnen wir ab. Wir werden uns dafür einsetzen, durch eine Verfassungsänderung solche Entscheidungen an eine 2/3-Mehrheit des Bundestages zu binden.

Die Bundeswehr muss mit begrenzten, aber qualitativ hochwertigen Beiträgen zur Stärkung der Zielsetzung und Fähigkeiten der Vereinten Nationen im Rahmen internationaler Friedenssicherung beitragen können. Dieses erfordert, gerade auch unter dem Eindruck der Anschläge vom 11. September
2001, eine Weiterentwicklung der bisherigen Reform der Bundeswehr. Wir wollen eine verkleinerte, moderne Bundeswehr mit maximal 200.000 SoldatInnen und die Abschaffung der Wehrpflicht.

Die Weizsäcker-Kommission hat mit ihrem Vorschlag einen konstruktiven Beitrag zur Debatte um die Bundeswehrreform geleistet. Die Wehrpflicht ist sicherheitspolitisch nicht mehr legitimierbar und blockiert eine finanzierbare Bundeswehrreform.

Wir sind für die Anpassung der Inneren Führung und des Leitbildes vom »Staatsbürger in Uniform« an die neuen Aufgaben der Bundeswehr und die Bedürfnisse einer Freiwilligenarmee, damit die Integration in die Gesellschaft und die demokratische Kontrolle weiterhin gewährleistet ist. Unser Ziel ist der deutliche Abbau militärischer Potentiale in unserem Land.

Wir wollen schrittweise die finanziellen Mittel zur Förderung der Entwicklungszusammenarbeit, der Friedens- und Konfliktforschung, vielfältiger Dialoginitiativen, der Ausbildung von Polizeikräften für internationale Friedensmissionen und des Zivilen Friedensdienstes spürbar erhöhen. (...)

\section{Abrüstung vorantreiben}

Wir wenden uns gegen einen neuen Rüstungswettlauf. Wir wollen eine aktive Abrüstungspolitik, die auf den bereits erreichten und vertraglich fixierten Erfolgen aufbaut und Antworten auf neue bzw. neu erkannte Bedrohungen formuliert. Diese reichen von der Kontrolle und Vernichtung konventioneller Kleinwaffen und Landminen bis hin zur Zerstörung von Massenvernichtungswaffen und der drastischen Reduzierung der Nuklearpotentiale. Die Verträge zur Nicht-Weiterverbreitung von Massenvernichtungswaffen, das Chemiewaffenübereinkommen, das B-Waffen-Übereinkommen und das Regime der Nichtverbreitung von Trägerwaffentechnologie wollen wir wirkungsvoller gestalten und stärken. Wir wollen Rüstungsexporte weiter begrenzen und Entscheidungsverfahren transparent gestalten. Wir setzen uns für einen regelmäßigen Bericht zur Rüstungsfolgenabschätzung ein. Abrüstung und Bundeswehrstrukturreform machen auch Konzepte der zivilen Umwandlung (Konversion) bisheriger militärischer Einrichtungen erforderlich; dazu müssen auf allen Ebenen angemessene Mittel bereitgestellt werden.

Quelle: http://www.gruen-wirkt.de/rsvgn/rs_dok/0,,7780,00. htm.

\section{Aus dem Wahlprogramm der FDP 2002-2006}

\section{Politik für eine freie Welt}

Liberale Außenpolitik ist werteorientierte Interessenpolitik. Deutschland muss eine aktive, verantwortungsvolle und angemessene Rolle in der Weltpolitik übernehmen; dabei muss es in seiner internationalen Verantwortung zwei Fehler vermeiden: es sollte sich nicht größer machen, als es ist, aber auch nicht kleiner. Deutschland sollte zusammen mit Partnern den Beitrag leisten, der seinem Gewicht und seinen Fähigkeiten entspricht und den auch seine Verbündeten von ihm erwarten. Die Prinzipien und Werte, die für unsere Verfassung und unser innerstaatliches Handeln gelten, sind auch für unsere Außenund Sicherheitspolitik verbindlich. 
Die internationale Verantwortung Deutschlands muss Hand in Hand gehen mit deutscher Außen- und Sicherheitspolitik in und mit der Europäischen Union. Die Notwendigkeit, die Handlungsfähigkeit und Effizienz der EU zu stärken, liegt mehr denn je auf der Hand. Deswegen sind die konstitutionelle Entwicklung und der erfolgreiche Abschluss der nächsten Erweiterungsrunde strategisch zentrale Felder für die deutsche Politik. Die europäische Integration, der Aufbau einer europäischen Sicherheits- und Friedensordnung, sowie die Stärkung der Vereinten Nationen liegen ebenso im deutschen Interesse wie der Export von Stabilität durch eine aktive Entwicklungs- und Menschenrechtspolitik und die Schaffung von Rahmenbedingungen für einen freien Welthandel.

Die Partnerschaft mit den Vereinigten Staaten von Amerika bleibt ein tragendes Fundament deutscher Außenpolitik. Zunehmende sicherheits- und handelspolitische Herausforderungen stellen die transatlantischen Beziehungen jedoch vor neue Bewährungsproben. Dabei wissen wir, dass der selbstbewusste Umgang miteinander letztlich auch Ausdruck unserer transatlantischen Wertegemeinschaft ist. Echte Partnerschaft wird durch Kritik nicht geschwächt, sondern gefestigt. Nicht aus dem Auge gelassen werden darf aber auch die Etablierung belastbarer, vertrauensvoller Beziehungen zu den östlichen Nachbarn, wie z. B. Russland und der Ukraine. Bei aller Notwendigkeit der Vertiefung der europäischen Integration und der euro-atlantischen Partnerschaft dürfen auch die traditionellen Beziehungen und die deutschen Interessen in Afrika, Lateinamerika und Asien nicht vernachlässigt werden.

\section{Europa ist unsere Antwort auf die internationalen Herausforderungen}

In den nächsten fünf Jahren stehen in der Europapolitik zentrale Aufgaben auf der Agenda: Europas Handlungsfähigkeit muss sichergestellt und die Vereinigung Europas vorangebracht werden. Im Einzelnen ist es dazu erforderlich, eine Verfassung für Europas Bürger zu erarbeiten, die europäische Sicherheitspolitik auszubauen, die europäische Innen- und Justizpolitik zu vertiefen, Europas Wirtschaft zu stärken und die europäische Agrar- und Strukturpolitik zu reformieren. Durch die Erfüllung dieser Aufgaben muss sich die Europäische Union zu einem starken und fairen Global Player entwickeln. Die FDP lehnt die Einführung eines Europaministers ab. Außen- und Europapolitik sind untrennbar und müssen im Interesse einer europäischen Politik Deutschlands im Auswärtigen Amt bleiben.

(...)

\section{Die europäische Außen- und Sicherheitspolitik ausbauen}

Europa muss außenpolitisch mit einer Stimme sprechen und in der Lage sein, in Konfliktfällen schnell und flexibel zu reagieren. Ziel der GASP ist es, durch effektive präventive Diplomatie Konflikte zu vermeiden und eine gesamteuropäische Friedens- und Stabilitätsgemeinschaft aufzubauen. Grundlage ist dabei eine den Menschenrechten und der Demokratie, der sozialen und ökologischen Marktwirtschaft sowie eine dem Ausgleich zwischen Süd und Nord verpflichtete Politik. Die FDP streitet für eine werteorientierte europäische Außenpolitik.

Die europäische Sicherheits- und Verteidigungspolitik (ESVP) muss konsequent ausgebaut und gefestigt werden. Ein Konkurrenzverhältnis zur Nordatlantischen Allianz, die auch künf- tig Motor und Garant einer breit angelegten europäischen Sicherheitsarchitektur bleiben muss, darf dabei nicht entstehen. Vielmehr ist eine strategische Partnerschaft zwischen EU und NATO bei der Krisenbewältigung das Ziel, in der die EU nur dann tätig wird, wenn sich die NATO nicht engagieren kann oder will. Die Einsatzkräfte der ESVP werden ab 2003 nationale Alleingänge überflüssig machen. Eine funktionierende ESVP stärkt nicht nur Europa, sondern auch die Allianz. Längerfristiges Ziel ist die Schaffung gemeinsamer europäischer Streitkräfte unter einheitlichem Oberbefehl. (...)

\section{Die Vereinten Nationen umfassend stärken}

Die Vereinten Nationen (VN) sind als zentrales weltinnenpolitisches Instrument alternativlos. Die FDP fordert, dass das VN-System in allen seinen Bereichen ausgebaut und nachhaltig gestärkt wird. Als drittgrößter Beitragszahler steht Deutschland hier in besonderer Verantwortung. Deutschland muss daher gemeinsam mit unseren Partnern in der Europäischen Union eine Initiative zur Stärkung der Vereinten Nationen als zentralem Instrument zur Bewältigung der neuen globalen Herausforderungen ergreifen.

Die FDP fordert eine zügige Reform der Vereinten Nationen. Hierzu zählt eine grundsätzliche Stärkung der Generalversammlung und des Generalsekretärs der VN, eine Neustrukturierung des Wirtschafts- und Sozialrates, wie sie die Weizsäcker-Quereshi-Kommission vorgeschlagen hat und eine Reform des Sicherheitsrates, die der Weltlage des 21. Jahrhunderts entspricht. Hierzu gehört die Beteiligung Deutschlands unter Aufrechterhaltung der Perspektive eines europäischen Sitzes ebenso wie einer angemessenen Beteiligung bislang nicht vertretener Weltregionen. Die entwicklungspolitischen und humanitären VN-Organisationen müssen zu wirkungsvollen Präventionsinstrumenten weiter entwickelt werden. Zur Stärkung friedenserhaltender und friedensschaffender Maßnahmen sind permanente VN-Kapazitäten aufzubauen. Deutschland soll sich für eine gemeinsame europäische VN-Politik durch Erarbeitung gemeinschaftlicher Positionen im Rahmen der VN-Generalversammlung einsetzen. (...)

\section{$\ddot{A} u ß e r e$ Sicherheit schafft Sicherheit auch im Inneren}

Frieden und Freiheit gehören unteilbar zusammen. Sie sind im Interesse der Menschen herbeizuführen und zu sichern, notfalls auch mit militärischen Mitteln. Dieser Maxime der Atlantischen Allianz verdanken wir Deutschen unsere Einheit in Freiheit. Deshalb setzen wir uns dafür ein, dass Deutschland seine neue Rolle verantwortungsvoll annimmt und die Bundeswehr schnell und konsequent bündnisfähig macht.

\section{Die Bundeswehr bündnisfähiger machen}

Die sicherheitspolitische Lage hat sich grundlegend geändert. Nach dem Ende des Kalten Krieges, dem Zerfall der Sowjetunion und der Öffnung der NATO für neue Mitglieder hat sich eine massive militärische Kräfteverschiebung vollzogen. Das Atlantische Bündnis ist heute jedem denkbaren Gegner in konventioneller Hinsicht mindestens dreifach überlegen, ohne auch nur einen Reservisten einberufen zu müssen. Die Mehrheit der NATO-Mitgliedstaaten hat die Wehrpflicht ausgesetzt, eine Entscheidung, die auch schnellstens für die Bundeswehr getroffen werden sollte. Die Wehrpflicht ist 
sicherheitspolitisch nicht mehr zu begründen. Die Anforderungen an Streitkräfte sind heute mit dem traditionellen Bild der Massenarmee nicht zu bewältigen.

Deutschland benötigt eine hochmotivierte, sehr gut ausgebildete und mit modernster Bewaffnung ausgerüstete Bundeswehr. Sie muss professionell, flexibel und schnell einsetzbar sein sowohl im Rahmen der Bündnisverteidigung, der Krisenbewältigung, der Terrorbekämpfung, der Friedenssicherung, als auch der humanitären und der Katastrophenhilfe.

(...)

Quelle: http://www.fdp-bundesverband.de/buergerprog.phtml.

\section{Aus dem Wahlprogramm der PDS 2002-2006}

Globale Probleme friedlich, kooperativ und gerecht lösen

»Frieden, Freiheit, Gerechtigkeit, Menschenrechte und eine Leben in Würde « - diesen Zielen muss deutsche Außenpolitik verpflichtet sein. Frieden kann nur wachsen, wo Gerechtigkeit herrscht. Freiheit gibt es nur dort, wo die Menschen ohne Angst vor Gewalt und ohne Sorge um das tägliche Brot leben können. Die dauerhafte Sicherung von Frieden und Freiheit verlangt daher kategorisch, die in der Welt von heute herrschenden Gegensätze zwischen Reich und Arm, Nord und Süd, Starken und Schwachen zu überwinden. Deutsche Außenpolitik soll durch eigenes Handeln, durch entsprechendes Wirken in der Europäischen Union, in Kooperation mit anderen Staaten und in der UNO dazu einen Beitrag leisten.

Für die anderen Parteien im Deutschen Bundestag zeigt sich die wiedergewonnene staatliche Souveränität Deutschlands an der Bereitschaft und Fähigkeit, Krieg führen und mit ihm als Normalität leben zu können. Erstmals seit dem Zweiten Weltkrieg wurden deutsche Soldaten, und zwar entgegen dem Verfassungsgebot und out of area, in den Krieg geschickt, Streitkräfte gelten wieder als Mittel zur Durchsetzung außenpolitischer Interessen. Damit finden wir uns nicht ab.

Deutschland zählt heute zu den stärksten Nationen in Europa mit weltpolitischem Gewicht. Wir halten an der Kultur militärischer und machtpolitischer Zurückhaltung fest, die lange Zeit die deutsche Nachkriegsgeschichte prägte. Die PDS hat im Bundestag aus guten Gründen die Beteiligung der Bundeswehr an Kriegen und Militärinterventionen abgelehnt und den Abzug dieser Militärkontingente aus den Einsatzgebieten gefordert. Dies wird so bleiben. Die PDS will Konflikte zivil und nicht militärisch lösen. Deutschland muss sich in der Welt als fairer Partner für globale Gerechtigkeit, nachhaltige Entwicklung und Wohlstand erweisen. Gerade als weltwirtschaftlich eng verflochtenes Land muss Deutschland an einer friedlichen, überlebensfähigen, sich nachhaltig entwickelnden Welt aktiv mitarbeiten. Dies bedeutet für uns auch, dass Deutschland seine Mitgliedschaft in der EU zur Durchsetzung einer sozialen und ökologischen Politik nutzt.

Auf internationaler Ebene treten wir für die Stärkung der internationalen Institutionen wie UNO und OSZE und für eine zuverlässige Einbindung Deutschlands in diese Institutionen ein. Deutschlands Zukunft liegt in einem geeinten Europa, einem Europa, das hegemoniale Ansprüche anderer zurückweist ohne eigene Vorherrschaftsansprüche zu stellen, einem Europa, das seine sozialstaatlichen Traditionen weiterentwickelt und sich für eine gerechte Weltordnung stark macht.
Das Völkerrecht verteidigen, die Vereinten Nationen stärken und demokratisieren

Ein Umsteuern in der internationalen Politik ist unumgänglich, wenn die Gewalt in der Welt verringert werden soll. Seit Jahrzehnten ungelöste Konflikte wie in Nahost, Südasien oder Afrika, die immer wieder zu eskalieren drohen, müssen endlich gerecht gelöst werden. Dies braucht neue Leitbilder und Regulationsformen für die friedliche Koexistenz und die gemeinsame Entwicklung.

Unter der Führung der USA wurde nach den schändlichen Terrorattacken vom 11. September 2001 der langandauernde Feldzug gegen »das Böse« ausgerufen. Wir haben uns strikt dagegen gewandt, dass auf Terror mit Krieg geantwortet wird. Ebenso wenden wir uns dagegen, die Terrorismusbekämpfung als Vorwand zu nutzen, um strategische und ökonomische Interessen durchzusetzen, wie dies die NATO und die USAdministration im Besonderen tun. Die Zivilisation muss zivilisiert verteidigt werden.

Die PDS verurteilt jeglichen Terrorismus, unabhängig davon, von wem er ausgeht und mit welchen Motiven er begründet wird. Wir bleiben dabei, dass Terroristen effizient verfolgt und der internationalen Strafgerichtsbarkeit zugeführt werden müssen und dass man ihre Finanzquellen - auch durch die Drosselung der internationalen Waffenmärkte - trocken legen muss. Eine Schlüsselrolle bei der Bekämpfung des internationalen Terrorismus müssen die Vereinten Nationen spielen. Die Verabschiedung einer umfassenden Konvention, die Einrichtung eines Zentrums für Terrorismusbekämpfung und der Aufbau von Instrumenten der Strafverfolgung gehören dazu. Die zügige Einrichtung des Internationalen Strafgerichtshofes sollte als vordringliche Aufgabe angesehen werden.

Wir wollen eine Welt, in der die wichtigsten Entscheidungen in der UNO und nicht in sich selbst mandatierenden Militärbündnissen, wie der NATO, oder in Organisationen, wie der G7/G8-Gruppe, getroffen werden. Die internationale Ordnung kann dauerhaft nur auf der Herrschaft des Rechts und nicht auf der Macht des Stärkeren beruhen. Die Vereinten Nationen brauchen endlich die Mittel, die sie zur Wahrung und Entwicklung einer friedlicheren Welt benötigen: Kompetenzen, Finanzen, Personal. Die Stärkung und Reform der Vereinten Nationen darf nicht auf die lange Bank geschoben werden. Es liegt im Interesse einer stabileren Weltordnung, dass die Stimme der sogenannten Dritten Welt in der Völkergemeinschaft lauter wird. Dies muss sich im Gewicht der UN-Vollversammlung und in der Sitzverteilung im Sicherheitsrat widerspiegeln. Die Vereinten Nationen müssen eine neue Verantwortung auch in der Entwicklung einer globalen Wirtschafts-, Sozial- und Umweltpolitik bekommen. Dazu gehört, dass sich der Internationale Währungsfond und die Weltbank in die UNO einordnen und an den Zielen der Armutsbekämpfung, einer gesamtgesellschaftlichen Entwicklungsförderung und der Durchsetzung der Menschenrechte orientieren müssen. 


\section{Abrüstung jetzt}

Nach einer kurzen Unterbrechung Anfang der 90er Jahre steigen die Rüstungsausgaben wieder - auch in der Bundesrepublik. Bei der weltweiten Ächtung, Kontrolle und Vernichtung von atomaren, biologischen und chemischen Massenvernichtungswaffen drohen neue Rückschläge. Die USA forcieren den Aufbau einer neuen Raketenabwehr-Rüstung, die mehr Unsicherheit bringt.

Das neue strategische Konzept der NATO ist auf weltweite Intervention zur Durchsetzung strategischer Interessen - Ö1, Gas, Wasser, zur Abwehr von Fluchtbewegungen und zur Sicherung von Handelswegen - ausgelegt. Aus einem Verteidigungsbündnis wurde ein Interventionsbündnis. Militärische Konfliktlösungen werden mehr und mehr zur Normalität. Doch es gibt Alternativen zur Militärlogik.

Die Logik des Friedens beruht auf vorausschauender Politik, auf wirtschaftlichem und sozialem Interessenausgleich, auf dem Ausbau ziviler Instrumente der Konfliktbewältigung, auf der intensiven Förderung des Dialogs der Kulturen. Sie zielt auf die Überwindung militärischer Strukturen und Instrumente. Ein Deutschland ohne Bundeswehr sowie eine Welt ohne Krieg sind und bleiben Ziel der Politik der PDS.

Dieser Logik zum Durchbruch zu verhelfen, ist eine langfristige Aufgabe, die unverzüglich begonnen werden muss.

Die Ernsthaftigkeit vorbeugender und nachhaltiger Konfliktbearbeitung muss sich an Haushaltszahlen messen lassen. Die Mittel für zivile Konfliktlösungen, darunter für den zivilen Friedensdienst und die Friedens- und Konfliktforschung, müssen kräftig angehoben werden. Wir wollen, dass das Militärbündnis NATO aufgelöst und durch kollektive Sicherheitsstrukturen im Rahmen der UNO und ihrer Regionalorganisationen, wie der OSZE, ersetzt wird. Unmittelbar treten wir dafür ein, dass die Bundesrepublik ihre Beteiligung am neuen strategischen Konzept der NATO aufkündigt, ihre Unterstützung für das gegenwärtige Auf- und Umrüstungsprogramm der NATO zurückzieht und auf der Aufhebung der nuklearen Erstschlagsdoktrinen besteht. Neuen Erweiterungsrunden der Allianz werden wir nicht zustimmen.

Wir halten an unseren Vorschlägen für eine mittelfristige Bundeswehrreform fest. 100.000 Soldaten und Soldatinnen sind mehr als ausreichend, um den grundgesetzlichen Auftrag der Landesverteidigung zu erfüllen. Da die Bundesrepublik nur noch von Freunden und Partnern umgeben ist, bedeuten einseitige Abrüstungsschritte nicht weniger, sondern mehr Sicherheit. Wir wollen eine aus Zeitsoldaten zusammengesetzte Freiwilligen-Armee anstelle der allgemeinen Wehrpflicht. Wir fordern einen Stopp der umfangreichen Modernisierungsund Beschaffungsprogramme, mit denen die Bundeswehr für weltweite Militäreinsätze fit gemacht werden soll. Die Personalstärke der Bundeswehr soll schrittweise reduziert werden, die frei werdenden Mittel im Wehretat sollen zu $80 \%$ in einen Konversionsfonds des Bundes überführt werden, um in den Standort-Regionen den sozial- und umweltverträglichen Übergang von militärischen zu zivilen Arbeitsund Sozialstrukturen zu ermöglichen. Ein Fünftel soll in internationale Kooperations- und Entwicklungsprojekte des Bundes fließen. Um diesen Umstellungsprozess zu organisieren, ist ein Bundesamt für Rüstungskonversion und Abrüstung unumgänglich.

Wir werden weiter darauf drängen, dass Rüstungsexporte verboten werden und dass in der nächsten Legislaturperiode eine deutliche Reduzierung bei den Waffenausfuhren erreicht wird.

(...)

Quelle: http://www.pds2002.de/positionen/programm/teil06.htm.

\section{Nachrichten - mit und ohne Kanten}

\begin{abstract}
- »Superministerium«: Für die meisten Politiker in Washington kam Bushs Ankündigung, »die größte Umstrukturierung in der US-Administration seit 55 Jahren« vornehmen $\mathrm{zu}$ wollen, wie ein Blitz aus heiterem Himmel. Ohne die Parlamentarie vorher informiert zu haben, wandte sich der US-Präsident via Fernsehen an das Volk und erklärte die Schaffung eines »Ministeriums zum Schutze der Heimat « (das Abendblatt berichtete).
\end{abstract}

Die neue Superbehörde soll nach dem Verteidigungsministerium das zweitgrößte Ministerium der USRegierung werden. Hauptaufgabe der neuen Behörde wird es sein, »die
Effektivität von Sicherheitsstellen zu erhöhen und den Schutz vor Terroranschlägen zu verbessern«.

Dafür sollen keine neuen Beamten eingestellt werden, sondern nur einzelne Abteilungen aus insgesamt acht Ministerien und mehr als 100 Behörden ausgegliedert werden. Nach den Berechnungen des Weißen Hauses soll das neue Ministerium über 169154 Angestellte und einen Jahreshaushalt von 37,45 Milliarden Dollar verfügen. So würden in Zukunft zahlreiche Organisationen wie Zoll, Küstenwache, Einwanderungsbehörde, Grenzkontrolle und das Bundesamt für Katastrophenschutz eng zusammenarbeiten. Auch die Bundespolizei
FBI sowie die Geheimdienste CIA und NSA müssten ihre Erkenntnisse an das Ministerium für Heimatschutz weiterleiten, würden ihre Unabhängigkeit allerdings behalten.

Während Politiker aller US-Parteien den überraschenden Schritt von George W. Bush grundsätzlich begrüßten, gab es von Seiten der Demokraten vereinzelte Kritik über den Zeitpunkt. Die Ankündigung kam, während die Spitzen von FBI und CIA vor einem parlamentarischen Untersuchungsausschuss eingestehen mussten, dass es innerhalb ihrer Organisationen im Vorfeld der Anschläge des 11. September, »gravierende Kommunikationsprobleme« gegeben habe. Der demo- kratische Senator Patrick Leahy, der Vorsitzende des Untersuchungsausschusses, spekulierte, ob die BushAdministration durch diese Ankündigung »von ihren Versäumnissen im Zusammenhang mit den Anschlägen des 11. September ablenken will«. Unter Experten wird Bushs Schritt als politische Niederlage angesehen, da die Opposition bereits kurz nach den Anschlägen solch ein Ministerium gefordert hatte.

Die Kritik an den massiven Kommunikationslücken zwischen FBI, CIA und NSA zwang Bush zum raschen Handeln. Angesichts der Parlamentswahlen in fünf Monaten will der Präsident einen Skandal verhindern. 